\title{
SENSITIVE DEPENDENCE FOR NONAUTONOMOUS DISCRETE DYNAMICAL SYSTEMS
}

\author{
ALEJANDRO MIRALLES, MARINA MURILLO-ARCILA, AND MANUEL SANCHIS
}

\begin{abstract}
Given a nonautonomous discrete dynamical system (NDS) $\left(X, f_{1, \infty}\right)$ we show that transitivity and density of periodic points do not imply sensitivity in general, i.e., in the definition of Devaney chaos there are no redundant conditions for NDS. In addition, we show that if we also assume uniform convergence of the sequence $\left(f_{n}\right)$ that induces the NDS, then sensitivity follows. Furthermore, in contrast to the autonomous case, we show that there exist minimal NDS which are neither equicontinuous nor sensitive.
\end{abstract}

\section{INTRODUCTION}

Nonautonomous discrete dynamical systems (NDS for short) were introduced by S. Kolyada and L. Snoha in [11]. A very readable account of some recent developments on the theory of nonautonomous discrete dynamical systems has been given in [3].

Let $(X, d)$ a metric space. An NDS is a pair $\left(X, f_{1, \infty}\right)$ where $f_{1, \infty}=\left(f_{n}\right.$ : $X \rightarrow X)_{n \in \mathbb{N}}$ is a sequence of continuous functions. The composition

$$
f_{1}^{n}:=f_{n} \circ f_{n-1} \circ \cdots \circ f_{2} \circ f_{1}
$$

is said to be the $n^{\text {th }}$-iterate of $f_{1, \infty}$ for all $n \in \mathbb{N}$ and, as usual, the symbol $f_{1}^{0}$ will stand for $I d_{X}$, the identity map on $X$. The orbit of a point $x \in X$ is the set

$$
\mathcal{O}_{f_{1, \infty}}(x):=\left\{x, f_{1}^{1}(x), f_{1}^{2}(x), \cdots f_{1}^{n}(x), \cdots\right\}
$$

which can also be described by the difference equation $x_{1}=x$ and $x_{n+1}=$ $f_{n}\left(x_{n}\right)$ for each $n \in \mathbb{N}$.

Recall that given a compact metric space $(X, d)$ and a sequence of continuous functions $\left(f_{n}: X \rightarrow X\right)_{n \in \mathbb{N}}$, a general form of a nonautonomous difference equation is the following:

2010 Mathematics Subject Classification. 47A16.

Key words and phrases. Non-autonomous systems, dynamical systems, sensitive dependence, equicontinuity.

The first author was partially supported by Spanish Government (MTM2014-53241P), Universitat Jaume I (P1-1B2014-35) and Generalitat Valenciana (AICO/2016/030). The second author was supported by Spanish Government (MTM 2016-75963-P). The third author was supported by Spanish Government (MTM2016-77143-P and MTM201564373P) and Generalitat Valenciana (AICO/2016/030). 


$$
\left\{\begin{array}{l}
x_{0}=x, \\
x_{n+1}=f_{n}\left(x_{n}\right)
\end{array}\right.
$$

for each $x \in X$. This kind of nonautonomous difference equation has been considered by several mathematicians (see for instance [17], [18] among others). Classical examples deal with $X=[0,1]$ the unit interval endowed with the usual metric.

NDS generalize autonomous discrete systems (ADS for short). An ADS is given by $(X, f)$, where $(X, d)$ is a metric space and $f: X \rightarrow X$ is continuous. These systems can be seen as a particular case of NDS just by considering $f_{n}=f$ for all $n \in \mathbb{N}$.

In this paper we address some questions related to transitivity and sensitivity on NDS. Our motivation is Devaney's definition of chaos [7]. Devaney defined an $\operatorname{ADS}(X, f)$ to be chaotic if it satisfies the following three conditions: (i) $(X, f)$ is transitive, (ii) the set of periodic points of $(X, f)$ is a dense subset of $X$ and (iii) $(X, f)$ is sensitive. Banks et al. [5] proved that conditions (i) and (ii) imply condition (iii) (to avoid degenerate cases $X$ is assumed to be infinite).

These results suggest the question whether the two former conditions, i.e. transitivity and density of periodic points, in an NDS imply the last one, i.e. sensitivity. This was proposed by Lan [14] in full generality:

In nonautonomous dynamical systems, does transitivity together with density of periodic points imply sensitivity?

Under additional conditions, considering the density of $k$-periodic points of $X$, Zhu et al. [18, Theorems 3.1 and 3.2] answered this question positively. However, in [16, Example 4.4] the authors provided a negative answer for an NDS on the interval [0,1]. In the papers by Zhu et al. [18], Lan [14] and Sanchez et al. [16] the authors considered a periodic point for the NDS to be $x \in X$ satisfying $f_{1}^{n}(x)=x$ for some $n \in \mathbb{N}$. In our paper we will consider periodic points to be those $x \in X$ satisfying that $f_{1}^{n k}(x)=x$ for some $n \in \mathbb{N}$ and any $k \in \mathbb{N}$. This definition coincides with the classical one of periodic point in the ADS case.

The paper is organized as follows. Section 2 is devoted to Devaney's chaos for NDS. We will provide new examples which give a negative answer to the question proposed by Lan [14]. Indeed, for each transitive non-sensitive ADS we show the existence of an NDS $\left(X, f_{1, \infty}\right)$ which is transitive, has a dense subset of periodic points but fails to be sensitive.

The main result of this section gives a positive answer to Lan's question and states that if $(X, d)$ is a metric space without isolated points and $\left(X, f_{1, \infty}\right)$ is an NDS such that $f_{n}: X \rightarrow X$ converges to $f$ uniformly, then transitivity of the NDS and density of periodic points in $X$ imply sensitivity.

In Section 3 we deal with equicontinuity and sensitivity. We show that if a transitive $\operatorname{NDS}\left(X, f_{1, \infty}\right)$ is equicontinuous at $x_{0}$, then $x_{0}$ is a transitive 
point. As a consequence, if an $\operatorname{NDS}\left(X, f_{1, \infty}\right)$ is topologically transitive and equicontinuous, then it is minimal. We finish with an example of a minimal NDS which is neither equicontinuous nor sensitive. This fact is a significant difference between the theory of ADS and the theory of NDS. Further references in ADS can be found in [9]. In [2,3] the reader can find further information about NDS. The interested reader in chaos, transitivity and sensitivity for NDS might consult $[4,8,10,15]$.

\section{Devaney chaos on NDS}

An $\operatorname{NDS}\left(X, f_{1, \infty}\right)$ is said to be topologically transitive (transitive for short) if for any pair of non-empty open sets $U, V \subset X$ there exists $N>0$ such that $f_{1}^{N}(U) \cap V \neq \emptyset$. It is said to have sensitive dependence on initial conditions (sensitive for short) if there exists a constant $\delta>0$ such that for any $x \in X$ and $\epsilon>0$ there exists $y \in X$ with $d(x, y)<\epsilon$ such that $d\left(f_{1}^{n}(x), f_{1}^{n}(y)\right)>\delta$ for some $n \geq 0$.

We will say that a point $p$ is periodic for the NDS $\left(X, f_{1, \infty}\right)$ if there exists $n \in \mathbb{N}$ such that $f_{1}^{n k}(p)=p$ for any $k \in \mathbb{N}$.

Definition 2.1. An $N D S\left(X, f_{1, \infty}\right)$ is Devaney chaotic if it satisfies the following conditions:

i) $\left(X, f_{1, \infty}\right)$ is topologically transitive;

ii) $\left(X, f_{1, \infty}\right)$ has a dense set of periodic points;

iii) $\left(X, f_{1, \infty}\right)$ has sensitive dependence on initial conditions.

As we have mentioned in Section 1, ADS are particular cases of NDS. Indeed, all these definitions can also be considered for ADS by considering $f_{n}=f$ for all $n \in \mathbb{N}$. In particular, $x \in X$ is periodic for the $\operatorname{ADS}(X, f)$ if $f^{n}(x)=x$ for some $n \in \mathbb{N}$. This shows that the definition of a periodic point for NDS coincides with the classical one for ADS. We will now show that sensitivity for NDS does not follow from transitivity and density of periodic points even if all the points are periodic. We first recall the following wellknown result.

Proposition 2.2. There exist ADS which are transitive but they fail to be sensitive.

Proof. It is sufficient to consider the $\operatorname{ADS}(\mathbb{T}, f)$ where $\mathbb{T}=\{z \in \mathbb{C}:|z|=1\}$ and $f$ is an irrational rotation, that is, $f: \mathbb{T} \longrightarrow \mathbb{T}$ is given by $f(z)=e^{i \alpha} z$ for $\alpha \in \mathbb{R} \backslash \mathbb{Q}$. It is well-known that the autonomous system $(\mathbb{T}, f)$ is topologically transitive [9, Example 1.12] but it fails to be sensitive since $d\left(f^{n}(z), f^{n}(w)\right)=d(z, w)$.

Hence we obtain the main result of this section.

Proposition 2.3. For any transitive non-sensitive $A D S(X, f)$ there exists an $\operatorname{NDS}\left(X, f_{1, \infty}\right)$ which is transitive, all its points are periodic but fails to be sensitive. 
Proof. Let $(X, f)$ be a topologically transitive ADS that fails to be sensitive which exists by Proposition 2.2. Suppose that $f$ has inverse $f^{-1}$. Denote by $f^{-n}$ the $n^{\text {th }}$-iterate $\overbrace{f^{-1} \circ \cdots \circ f^{-1}}^{n-f \text { fold }}$ and define the NDS $\left(X, f_{1, \infty}\right)$ where the sequence $\left(f_{n}\right)$ is given by

$$
\left(f_{1}, f_{2}, f_{3}, f_{4}, f_{5}, f_{6}, f_{7}, \ldots\right)=\left(f, f^{-1}, f^{2}, f^{-2}, f^{3}, f^{-3}, \ldots\right)
$$

so that the sequence

$$
\left(f_{1}^{1}, f_{1}^{2}, f_{1}^{3}, f_{1}^{4}, f_{1}^{5}, f_{1}^{6}, f_{1}^{7}, \ldots\right)=\left(f, I d_{X}, f^{2}, I d_{X}, f^{3}, I d_{X}, \ldots\right) .
$$

It is clear that every $x \in X$ is periodic since $f_{1}^{2 k}=I d_{X}$ for any $k \in \mathbb{N}$.

We show that the NDS is topologically transitive. Indeed, let $U, V \neq \emptyset$ be open sets of $X$. Since $(X, f)$ is topologically transitive, there exists $n$ such that $f^{n}(U) \cap V \neq \emptyset$. It is clear that there exists $m \in \mathbb{N}$ such that $f_{1}^{m}=f^{n}$ and we are done.

Now we show that $\left(X, f_{1, \infty}\right)$ is not sensitive. Since $(X, f)$ is not sensitive, for any $k \in \mathbb{N}$, there exists $x_{k} \in X$ and $0<\varepsilon_{k}<1 / k$ such that $\sup _{n \in \mathbb{N}} d\left(f^{n}\left(x_{k}\right), f^{n}(y)\right)<1 / k$ for all $y \in X$ satisfying $d\left(x_{k}, y\right)<\varepsilon_{k}$.

Notice that for any $n \in \mathbb{N}$ there exists $m_{n} \in \mathbb{N}_{0}$ such that $f_{1}^{m_{n}}=f_{n}$. So for any $k \in \mathbb{N}$, we have that

$$
\sup _{n \in \mathbb{N}} d\left(f_{1}^{m_{n}}\left(x_{k}\right), f_{1}^{m_{n}}(y)\right)=\sup _{n \in \mathbb{N}} d\left(f^{n}\left(x_{k}\right), f^{n}(y)\right)<\frac{1}{k}
$$

for any $y \in X$ such that $d\left(x_{k}, y\right)<\varepsilon_{k}$ so $\left(X, f_{1, \infty}\right)$ is not sensitive.

Although we provide a negative answer for the question proposed in [14], the answer becomes true if we assume an extra condition on the NDS.

Theorem 2.4. Let $(X, d)$ be a metric space without isolated points. Suppose that $f_{n}: X \rightarrow X$ converges uniformly to $f$. If

a) $\left(X, f_{1, \infty}\right)$ is topologically transitive and

b) there exists a dense subset $P \subset X$ of periodic points for $\left(X, f_{1, \infty}\right)$, then $\left(X, f_{1, \infty}\right)$ is sensitive.

In order to show this result we need the following lemmas.

Lemma 2.5. Let $(X, d)$ be a metric space without isolated points. Suppose that $f_{n}: X \rightarrow X$ converges pointwise to $f$. Then,

a) If $p$ is a periodic point for $\left(X, f_{1, \infty}\right)$ then $p$ is a periodic point for $(X, f)$.

b) If there exists an infinite set of periodic points of $f$, then there exists $\eta>0$ such that for any $x \in X$ there is a periodic point $p$ of $f$ such that $d\left(x, f^{n}(p)\right) \geq \eta$ for all $n \in \mathbb{N}$.

Proof. First we prove a). Since $p$ is periodic, there exists $N \in \mathbb{N}$ such that $f_{1}^{j N}(p)=p$ for all $j \in \mathbb{N}$. Notice that for any $0 \leq i \leq N$ we have that $f_{1}^{j N+i}(p)=f_{j N+i} \circ \cdots \circ f_{j N+1}(p)$ which is convergent to $f^{i}(p)$ when $j \rightarrow \infty$. 
In particular, for $i=N$, we have that $f_{1}^{j N+N}(p)=p$ is convergent to $f^{N}(p)$ so $f^{N}(p)=p$ and we are done.

Now we show b). Since $X$ has no isolated points it is an infinite set, then we can find two periodic points $p_{1}, p_{2}$ whose orbits are disjoint (since the orbits are finite). Hence,

$$
\eta:=\inf _{m, n \in \mathbb{N}_{0}} d\left(f^{m}\left(p_{1}\right), f^{n}\left(p_{2}\right)\right) / 2>0 .
$$

It then follows from the triangle inequality that, for any $x \in X$, either for $j=1$ or for $j=2$ we have that $d\left(x, f^{n}\left(p_{j}\right)\right) \geq \eta$ for all $n \in \mathbb{N}_{0}$.

For any pair of non-empty open sets $U$ and $V$ of $X$, let $N(U, V)=\{n \in$ $\left.\mathbb{N}: f_{1}^{n}(U) \cap V \neq \emptyset\right\}$.

Lemma 2.6. Let $(X, d)$ be a metric space without isolated points. If $\left(X, f_{1, \infty}\right)$ is topologically transitive, then for any pair of non-empty open subsets $U, V$ of $X$, the set $N(U, V)$ is infinite.

Proof. Let $U, V$ be non-empty open sets. Since the NDS is topologically transitive, $N(U, V)$ is non-empty. Let us suppose that it is finite and let

$$
m=\max \left\{n \in \mathbb{N}: f_{1}^{n}(U) \cap V \neq \emptyset\right\} .
$$

Since $X$ has no isolated points, $V$ contains infinitely many points, thus we can fix a collection $V_{j} \subset V, j=1, \ldots, m+1$ of mutually disjoint open sets. Since $m \in N(U, V)$, there exists $x \in U$ such that $f_{1}^{m}(x) \in V$. Now, there is $j_{0} \in\{1, \ldots, m+1\}$ such that $f_{1}^{i}(x) \notin V_{j_{0}}$ for all $i=1, \ldots, m$ due to the choice of $\left(V_{j}\right)_{j=1}^{m+1}$. By continuity, there is an open neighbourhood $U^{\prime}$ of $x$ contained in $U$ such that

$$
f_{1}^{i}(U) \cap V_{j_{0}}=\emptyset, \quad i=1, \ldots, m .
$$

Since $\left(X, f_{1, \infty}\right)$ is transitive, there exists $k \in \mathbb{N}$ such that $f_{1}^{k}\left(U^{\prime}\right) \cap V_{j_{0}} \neq \emptyset$. By (1), we have $k>m$ which is a contradiction because $N\left(U^{\prime}, V_{j_{0}}\right) \subset$ $N(U, V)$.

We now provide the proof of our main theorem.

Proof of Theorem 2.4. We fix a metric $d$ defining the topology of $X$.

Let $x \in X$ and $\varepsilon>0$. By Lemma 2.5, there is a periodic point $p$ such that

$$
d\left(x, f^{n}(p)\right) \geq \eta \quad \text { for } \quad n \in \mathbb{N}_{0} .
$$

We now claim that $\left(X, f_{1, \infty}\right)$ is sensitive with sensitivity constant $\delta:=\eta / 4$. By assumption there is a periodic point $q$ such that

$$
d(x, q)<\min \left\{\varepsilon, \frac{\eta}{4}\right\} .
$$


Let $q$ have period $N$, that is, $f_{1}^{j N}(q)=q$ for any $j \in \mathbb{N}$. Since the functions $f^{j}$ are continuous for $j=1,2, \cdots, N$, there is some neighbourhood $V$ of $p$ such that

$$
d\left(f^{j}(p), f^{j}(y)\right)<\delta / 2 \quad \text { for } \quad j=0,1, \ldots, N \quad \text { and } \quad y \in V .
$$

Consider $U=B_{\varepsilon}(x)$ and $V$ the neighbourhood of $p$. Since $f_{n}$ converges to $f$ uniformly, we find $j_{0} \in \mathbb{N}$ such that for any $j \geq j_{0}$ we have for any $0 \leq l<N$

$$
d\left(\left(f_{j N} \circ f_{j N-1} \circ \cdots \circ f_{(j-1) N+l+1}\right)(v), f^{N-l}(v)\right)<\delta / 2 \text { for any } v \in V .
$$

Since $\left(X, f_{1, \infty}\right)$ is topologically transitive, there exists an infinite set of positive integers $m$ such that $f_{1}^{m}(U) \cap V \neq \emptyset$ by Lemma 2.6. Hence we can consider $k=(j-1) N+i$ for $0 \leq i<N$ such that $j \geq j_{0}$ and $f_{1}^{k}(U) \cap V \neq \emptyset$. Take $z \in U$ (that is, $d(x, z)<\varepsilon$ ) such that $f_{1}^{k}(z) \in V$.

Let $S:=f_{j N} \circ f_{j N-1} \circ \cdots \circ f_{(j-1) N+i+1}$. By hypothesis we will obtain that

$$
d\left(S\left(f_{1}^{k}(z)\right), f^{N-i}\left(f_{1}^{k}(z)\right)\right)<\delta / 2 .
$$

By the triangle inequality we obtain using inequality (3) that

$$
\begin{gathered}
d\left(f^{N-i}(p), S\left(f_{1}^{k}(z)\right)\right) \leq \\
d\left(S\left(f_{1}^{k}(z)\right), f^{N-i}\left(f_{1}^{k}(z)\right)\right)+d\left(f^{N-i}(p), f^{N-i}\left(f_{1}^{k}(z)\right)\right)<\delta / 2+\delta / 2=\delta
\end{gathered}
$$

and together with (2) then yields that

$$
\begin{aligned}
d\left(f_{1}^{j N}(q), f_{1}^{j N}(z)\right)=d\left(f_{1}^{j N}(q), S\left(f_{1}^{k}(z)\right)\right)=d\left(q, S\left(f_{1}^{k}(z)\right)\right) & \geq \\
d\left(x, f^{N-i}(p)\right)-d\left(f^{N-i}(p), S\left(f_{1}^{k}(z)\right)\right)-d(x, q) & >4 \delta-\delta-\delta=2 \delta .
\end{aligned}
$$

Hence, either $d\left(f_{1}^{j N}(x), f_{1}^{j N}(q)\right)>\delta$ or $d\left(f_{1}^{j N}(x), f_{1}^{j N}(z)\right)>\delta$ and then the theorem holds.

\section{EquiCONTINUITY AND SENSITIVITY ON NDS}

An NDS $\left(X, f_{1, \infty}\right)$ is said to be equicontinuous at the point $x_{0}$ if for every $\varepsilon>0$ there exists $\delta>0$ such that $d\left(f_{1}^{n}(y), f_{1}^{n}\left(x_{0}\right)\right)<\varepsilon$ for all $n \geq 0$ whenever $d\left(y, x_{0}\right)<\delta$. An $\operatorname{NDS}\left(X, f_{1, \infty}\right)$ is said to be equicontinuous if it is equicontinuous at every point $x_{0} \in X$. It is a well-known fact that an equicontinuous NDS $\left(X, f_{1, \infty}\right)$ defined on a compact metric space $X$ is uniformly equicontinuous, that is, for every $\varepsilon>0$ there is $\delta>0$ such that $d\left(f_{1}^{n}(y), f_{1}^{n}(x)\right)<\varepsilon$ for all $n \geq 0$ whenever $d(y, x)<\delta$. Recall also that a point $x_{0} \in X$ is called transitive if the orbit of $x_{0}$ is dense in $X$. If all the points of $X$ are transitive, then the NDS is called minimal.

An interesting aspect of the theory of discrete dynamical systems lies in the fact that if a topologically transitive $\operatorname{ADS}(X, f)$ is equicontinuous at least at a point of $X$, then the set of equicontinuous points coincides with the set of transitive points. In particular, a minimal ADS is either equicontinuous or sensitive (see [1] for details and, for a generalization to semigroup actions, see [12]). As the example below shows, the situation is 
quite different if we replace ADS by NDS. We first prove an easy connection between equicontinuity and transitive points.

Theorem 3.1. Let $\left(X, f_{1, \infty}\right)$ be a topologically transitive $N D S$. If $\left(X, f_{1, \infty}\right)$ is equicontinuous at $x_{0}$, then $x_{0}$ is a transitive point.

Proof. Let $y \in X$ and $\varepsilon>0$. Since the NDS is equicontinuous at $x_{0}$, there exists an open ball $B_{\delta}\left(x_{0}\right)$ such that $d\left(f_{1}^{n}\left(x_{0}\right), f_{1}^{n}(z)\right)<\varepsilon$ for any $z \in B_{\delta}\left(x_{0}\right)$ and all $n \in \mathbb{N}$. Since $\left(X, f_{1, \infty}\right)$ is topologically transitive, we can find $n \geq 0$ such that $f_{1}^{n}\left(B_{\delta}\left(x_{0}\right)\right) \cap B_{\varepsilon}(y) \neq \emptyset$. Then, if $f_{1}^{n}(z) \in f_{1}^{n}\left(B_{\delta}\left(x_{0}\right)\right) \cap B_{\varepsilon}(y)$, we have

$$
d\left(f_{1}^{n}\left(x_{0}\right), y\right) \leq d\left(f_{1}^{n}\left(x_{0}\right), f_{1}^{n}(z)\right)+d\left(f_{1}^{n}(z), y\right)<2 \varepsilon,
$$

that is, $f_{1}^{n}\left(x_{0}\right)$ belongs to $B_{2 \varepsilon}(y)$. This completes the proof.

A straightforward consequence of the previous result is the following corollary.

Corollary 3.2. If an $N D S\left(X, f_{1, \infty}\right)$ is topologically transitive and equicontinuous, then $\left(X, f_{1, \infty}\right)$ is minimal.

We now present the promised result. Recall that the Cantor set $\mathcal{C}$ has minimal equicontinuous systems. Indeed, minimal equicontinuous systems on the Cantor set $\mathcal{C}$ are conjugate to an odometer, that is, they are Kronecker systems on $\mathcal{C}$ (see [13]) and, consequently, they are isometries. We will take advantage of the following useful property of the Cantor set: two clopen subsets of $\mathcal{C}$ are homeomorphic (this is a particular case of the well-known Brower's theorem [6] which states that every non-empty, perfect, compact, totally disconnected and metrizable space is homeomorphic to the Cantor set).

Theorem 3.3. There exists a minimal NDS which is neither equicontinuous nor sensitive.

Proof. Let $\left\{M_{1}, M_{2}\right\}$ be a partition of the Cantor set with $M_{i}$ a clopen subset for each $i=1,2$ and consider a minimal equicontinuous isometry $T$ on $\mathcal{C}$. Now, given the clopen sets $C_{i}=T\left(M_{i}\right)$ for each $i=1,2$, our first step is to define a sequence of homeomorphisms $\left\{f_{n}: n \in \mathbb{N}\right\}$ on $\mathcal{C}$ that will allow us to construct a non-equicontinuous NDS. For this, choose a point $x_{1}$ in $C_{2}$ and consider a sequence $\left\{F_{n}: n \in \mathbb{N}\right\}$ which satisfies, for each $n \in \mathbb{N}$, the following properties:

(1) $F_{n}$ is a clopen subset of $\mathcal{C}$ whose diameter is $1 / n$;

(2) $F_{n+1} \subsetneq F_{n}$;

(3) $x_{1} \in F_{n+1}$.

Now, choose clopen subsets $S_{1}, S_{2}$ of $C_{2}$ such that $d\left(S_{1}, S_{2}\right)=\varepsilon_{0}>0$. Select, for each $n \in \mathbb{N}$, homeomorphisms $g_{n}: C_{2} \backslash F_{n} \rightarrow C_{2} \backslash\left(S_{1} \cup S_{2}\right)$, $h_{n}: F_{n} \backslash F_{n+1} \rightarrow S_{1}$ and $q_{n}: F_{n+1} \rightarrow S_{2}$. Given $n \in \mathbb{N}$, we define $f_{n}$ as follows: 


$$
f_{n}(x)=\left\{\begin{array}{lll}
x & \text { if } \quad x \in C_{1}, \\
g_{n}(x) & \text { if } \quad x \in C_{2} \backslash F_{n}, \\
h_{n}(x) & \text { if } x \in F_{n} \backslash F_{n+1}, \\
q_{n}(x) & \text { if } \quad x \in F_{n+1}
\end{array}\right.
$$

Now let $\left(\mathcal{C}, f_{1, \infty}\right)$ be the NDS defined by the sequence

$$
\left\{T, f_{1}, f_{1}^{-1}, T, T^{-1}, f_{2}, f_{2}^{-1}, T^{2}, T^{-2}, f_{3}, f_{3}^{-1}, T^{3}, T^{-3}, \ldots\right\} .
$$

It is an easy matter to check that

$$
\begin{array}{ll}
f_{1}^{1}=T, & f_{1}^{2}=f_{1} \circ T \\
f_{1}^{3}=T, & f_{1}^{4}=T^{2} \\
f_{1}^{5}=T, & f_{1}^{6}=f_{2} \circ T \\
f_{1}^{7}=T, & f_{1}^{8}=T^{3} \\
f_{1}^{9}=T, & f_{1}^{10}=f_{3} \circ T
\end{array}
$$

Notice that the sequence $\left\{T^{n}: n \in \mathbb{N}\right\}$ is a subsequence of $\left\{f_{1}^{n}: n \in \mathbb{N}\right\}$. Thus, transitivity and minimality of $\left(\mathcal{C}, f_{1, \infty}\right)$ follow from transitivity and minimality of $(\mathcal{C}, T)$. Moreover, since the restriction of $f_{n}$ to $C_{1}$ is the identity for all $n \in \mathbb{N}$, the $\operatorname{NDS}\left(\mathcal{C}, f_{1, \infty}\right)$ is equicontinuous at every $x \in C_{1}$ (since $T$ is equicontinuous). Thus, $\left(\mathcal{C}, f_{1, \infty}\right)$ is nonsensitive.

We will prove that $\left(\mathcal{C}, f_{1, \infty}\right)$ is nonequicontinuous. Indeed, for fixed $\delta>0$, we can choose $n \in \mathbb{N}$ with $1 / n<\delta$. If $x \in F_{n} \backslash F_{n+1}$, then $f_{n}(x)=h_{n}(x) \in$ $S_{1}$. Since $f_{n}\left(x_{1}\right)=q_{n}\left(x_{1}\right) \in S_{2}$, we have

$$
d\left(( f _ { n } \circ T ) \left(T^{-1}(x),\left(f_{n} \circ T\right)\left(T^{-1}\left(x_{1}\right)\right) \geq \varepsilon_{0} .\right.\right.
$$

Since $T^{-1}$ is an isometry, this proves that $\left(\mathcal{C}, f_{1, \infty}\right)$ is not equicontinuous at $T^{-1}\left(x_{1}\right)$.

Remark 3.4. As the point $T^{-1}\left(x_{0}\right)$ of Theorem 3.3 shows, for transitive $N D S$ the existence of equicontinuous points does not imply that every transitive point is equicontinuous. This is an important difference with the case of $A D S[1]$.

Acknowledgements. The authors would like to thank Professor Alfred Peris for his fruitful suggestions concerning Lemma 2.6. 


\section{REFERENCES}

[1] E. Akin, J. Auslander and K. Berg, When is a transisitive map chaotic?, Convergence in ergodic theory and probability (Columbus, OH, 1993), 25-40, Ohio State Univ. Math. Res. Inst. Publ., 5, de Gruyter, Berlin, 1996.

[2] LL. Alseda, S. Kolyada, J. LLibre and L. Snoha, Entropy and periodic points for transitive maps, Trans. Amer. Math. Soc. 351(4) (1999), 1551-1573.

[3] F. Balibrea, T. Caraballo, P. D. Kloeden and J. Valero, Recent developments in dynamical systems: Three perspectives, Int. J. Bifurc. Chaos 20(9) (2010), 2591-2636.

[4] F. Balibrea and P. Oprocha, Weak mixing and chaos in nonautonomous discrete systems, App. Math. Lett. 25 (2012) 1135-1141.

[5] J. Banks, J. Brooks, G. Cairns, G. Davis and P. Stacey, On Devaney's definition of chaos, Amer. Math. Monthly 99 (1992), 332-334.

[6] L.E.J. Brouwer, On the structure of perfect sets of points, in KNAW, Proceedings, 12, 1909-1910, Amsterdam, 1910, 785-794.

[7] L. R. Devaney, An introduction to chaotic dynamical systems, Benjamin/Cummings, Menlo Park, CA, 1986; second edition, Addison-Wesley, Redwood City, CA, 1989.

[8] J. Dvorakova, Chaos in nonautonomous discrete dynamical systems, Commun. Nonlinear Sci. Numer. Simulat. 17 (2012), 4649-4652.

[9] K.-G. Grosse-Erdmann and A. Peris Manguillot. Linear chaos, Universitext, SpringerVerlag London Ltd., London, 2011.

[10] Q. Huang, Y. Shi and L. Zhang, Sensitivity of non-autonomous discrete dynamical systems, Appl. Math. Lett. 39 (2015), 31-34.

[11] S. Kolyada and L. Snoha, Topological entropy of nonautonomous dynamical systems, Random Comp. Dynam. 4(2-3) (1996), 205-233.

[12] E. Kontorovich and M. Megrelishvihi, A note on sensitive of semigroup actions, Semigroup Forum 76 (2008) 133-141.

[13] P. Kurka, Topological and symbolic dynamics, Cours Sépecialisés [Specialized Courses], 11, Société Mathématique de France, Paris, 2003.

[14] Y. Lan, Chaos in nonautonomous discrete fuzzy dynamical systems, J. Nonlinear Sci. Appl. 9 (2016), 404-412.

[15] L. Liu and Y. Sun, Weakly mixing sets and transitive sets for non-autonomous discrete systems, Adv. Difference Equ. (2014), 2014:17, 9 pp.

[16] I. Sánchez, M. Sanchis and H. Villanueva, Chaos in hyperspaces of nonautonomous discrete systems, Chaos, Solitons \& Fractals 94,(2017), 68-74.

[17] Y. Shi, Chaos in nonautonomous discrete dynamcial systems approached by their induced mappings, Int. J. Bifurc. Chaos 22(11) (2012), 1-12.

[18] H. Zhu, L. Liu and J. Wang, A note on stronger forms of sensitivity for inverse limit dynamical systems, Adv. Differ Equ 101 (2015), 1-9.

Institut Universitari de Matemàtiques i Aplicacions de Castelló (IMAC), Escuela Superior de Tecnología y Ciencias Experimentales, Universitat Jaume

I, Campus de Riu Sec, E-12071, Castelló de la Plana, Spain.

E-mail address: mirallea@uji.es

E-mail address: murillom@uji.es

E-mail address: sanchis@mat.uji.es 